\title{
An Examination of State and Federal Opioid Analgesic and Continuing Education Policies: 2016-2018
}

\author{
Kathryn Duensing (D) \\ Robert Twillman (D) ${ }^{1-3}$ \\ Stephen Ziegler ${ }^{4,5}$ \\ M Soledad Cepeda (iD) \\ David Kern $\mathbb{1 D}^{6}$ \\ Maribel Salas $\mathbb{D i D}^{7,8}$ \\ Gregory Wedin 9
}

'RKT Consulting, LLC, Lenexa, KS, USA; ${ }^{2}$ Behavioral Health Section, Saint Luke's Health System, Kansas City, MO, USA;

${ }^{3}$ Department of Psychiatry and

Behavioral Sciences, University of Kansas

School of Medicine, Kansas City, KS,

USA; ${ }^{4}$ Center for Effective Regulatory

Policy and Safe Access, Denver, CO, USA;

${ }^{5}$ College of Professional Studies, Purdue

University, Fort Wayne, IN, USA;

${ }^{6}$ Department of Epidemiology, Janssen

Research \& Development, Titusville, NJ,

USA; ${ }^{7}$ Department of Epidemiology,

Clinical Safety and Pharmacovigilance,

Daiichi Sankyo, Basking Ridge, NJ, USA;

${ }^{8}$ University of Pennsylvania Perelman

School of Medicine, Philadelphia, PA,

USA; ' Upsher-Smith Laboratories, LLC,

Pharmacovigilance and Risk Management,

Maple Grove, MN, USA
This article was published in the following Dove Press journal:

Journal of Pain Research

Introduction: Opioid overdose deaths in the United States have climbed sharply over the past two decades. Simultaneously, increased awareness of inadequately treated chronic pain has resulted in increased opioid analgesic prescribing. The correlation between these two phenomena has led policymakers to posit that they are causally linked, and to implement policy changes supporting safe opioid prescribing.

Purpose: To evaluate the impact of its Opioid Analgesic Risk Evaluation and Mitigation Strategy (REMS) program, the US Food and Drug Administration (FDA) requested the opioid manufacturers responsible for implementing that program provide information regarding opioid policy changes from 2016 to 2018. FDA also requested a survey of state requirements for pain and opioid prescribing continuing education (CE), the number of prescribers affected by those requirements, the extent to which a REMS-compliant CE program would meet each state's requirements, and the number of relevant $\mathrm{CE}$ programs available.

Results: Results indicate that 527 federal and state opioid-related policies (statutes, rules/ regulations, and guidelines) were approved during the 2016-2018 study period. While the largest number of these policies focused on prescription drug monitoring programs, 170 specifically imposed limits on opioid prescribing and an additional 35 specifically referred to, or incorporated, the Centers for Disease Control and Prevention opioid prescribing guideline. We also found that 46 states and the District of Columbia mandated some amount of pain or opioid prescribing CE for prescribers renewing their licenses. These mandates potentially affected as many as 1.7 million prescribers. In $69 \%$ of cases, a REMS-compliant CE program would fully meet the state mandates for various types of prescribers.

Conclusion: The severity and complexity of the problems of pain management and opioid overdose have led to large-scale intervention by policymakers. Assessing the impact of these changes is difficult, at best, but will be necessary if interventions are to be refined to increase their effectiveness.

Keywords: opioids, FDA blueprint, prescribing, law, policy, CDC, REMS

\section{Introduction}

The twin crises of undertreated pain and opioid overdose deaths pose major challenges to policymakers and communities. The US Centers for Disease Control and Prevention (CDC) has estimated that 50 million people are affected by chronic pain, and the number of opioid-related overdose deaths over a five-year period has increased from 25,052 in 2013 to 47,600 in 2017., ${ }^{1,2}$ Both public health problems are long-standing and have unique and complicated histories.
Correspondence: Robert Twillman Saint Luke's Health System, Kansas City, MO, USA

$\mathrm{Tel}+\mid$ 9|3-205-3746

Email Bob.Twillman@gmail.com
Journal of Pain Research 2020:13 2431-2442 
Twenty years ago, the United States Congress passed, and the president signed, the Pain Relief Promotion Act, a law that would "amend the Controlled Substances Act to promote pain management and palliative care." Congress recognized that while "the use of certain narcotics and other drugs" carries the potential for abuse, the inadequate treatment of pain "is a serious public health problem" and that "physicians should not hesitate to dispense or distribute controlled substances when medically indicated." Starting in 2001, the "Decade on Pain Control and Research" had begun. ${ }^{4}$

Today, some twenty years later, the United States continues to struggle with ensuring access to opioid analgesics when medically indicated while also preventing misuse, abuse, and overdose. As a nation, we continue to experience an alarming increase in opioid-related overdoses, ${ }^{5}$ and while most fatal overdoses now involve the use of illicit opioids, the misuse and abuse of prescription opioid analgesics continues to pose significant public health challenges. ${ }^{2}$ The severity of the problem has spurred policymakers, governmental agencies, and non-governmental organizations to address the overdose crisis in a variety of ways which have included the creation of new laws and regulations.

Governmental policy, whether in the form of a state or federal law, rule, regulation, agency decision, or guideline can impact the practice of medicine and the prescribing of drugs. ${ }^{6-9}$ For instance, the federal Controlled Substances Act of 1970 in conjunction with state laws and regulations concerning medical practice, determines who is authorized to prescribe controlled substances and under what circumstances. ${ }^{7}$ Moreover, because continuing education is one factor of many that can influence opioid prescribing and other aspects of pain treatment, states have experimented with a variety of educational policies, whether to make them voluntary or mandatory, ${ }^{10}$ and whether to require them at all. In 2016, Davis and Carr reported that only

five states (CT, IA, MD, SC, and TN), require all or nearly all physicians to obtain periodic CME on such topics as pain management, controlled substance prescribing, or substance use disorders. (p.102) $)^{11}$

However, a year later, $\mathrm{Xu}$ and colleagues reported a significant change in the $\mathrm{CE}$ landscape:

Pain management and controlled substance prescribing are the two most common subject specific $\mathrm{CE}$ requirements, with 29 states (57\%) having one or both requirements. All of these states, except Rhode Island and Wisconsin, established their requirements through statute (p.15) ${ }^{12}$

In response to an increase in morbidity and mortality associated with prescription drugs, Congress passed the Food and Drug Administration Amendments Act of 2007. The act enhanced FDA's authority and enabled it to require REMS for certain manufactured drugs, a drug safety program "designed to reinforce medication use behaviors and actions that support the safe use of that medication."13 REMS may involve the development of medication guides, Elements to Assure Safe Use, and/or a communication plan. Many REMS programs include educational activities in some form. In 2012, FDA required opioid analgesic (OA) manufacturers to implement a class-wide REMS for all extendedrelease and long-acting (ER/LA) opioid medications. A major element of this REMS was the inclusion of Accredited Continuing Education based on an FDA Blueprint that outlined the content for the educational activities. In 2018, this opioid REMS was further expanded to include immediate-release opioid analgesics, a program now known as the Opioid Analgesic REMS. In that same year, the FDA released an updated version of its educational blueprint, a roadmap to guide REMS-based continuing education across the United States, an intervention that is funded by REMS Program Companies (RPC) for all health care providers (HCPs) who prescribe or participate in the treatment and monitoring of patients who receive OAs, including pharmacists and nurses. ${ }^{14}$

In light of these changes, coupled with a study requested of RPC by FDA to help them better understand changes in OA prescribing patterns, this article reports a summary of major OA policy changes occurring from 2016 to 2018 at the state and federal levels and a survey of state CE requirements related to pain or opioid prescribing from September 2018 to July 2019. This article is derived from the RPC commissioned study noted above. To our knowledge, this article represents the first comprehensive survey of these topics.

\section{Materials and Methods Policy Adoption}

In late 2018, FDA requested that RPC provide information on governmental policies related to OA prescribing adopted during the period from 2016 to 2018. The following federal and state policies were included in our analysis: initiatives from the President of the United States and numerous 
Governors; federal and state statutes, rules, and regulations; state guidelines from licensing agencies and departments of health; State Medicaid program opioid policies; and federal guidelines and policies from the CDC, FDA, Department of Health and Human Services, Centers for Medicare \& Medicaid Services (CMS), Department of Veterans Affairs (VA), Department of Defense (DoD), and the National Academies of Sciences, Engineering, and Medicine (NASEM).

Legislative and regulatory policy data at the state and federal level for the three-year study period were extracted from a larger database of pain management-related policies, contemporaneously gathered by the staff of the Academy of Integrative Pain Management (author KD, supervised by author RT) using legislative tracking software (CQ Roll Call's StateTrack program) driven by key word searches; see Appendix A for details regarding the search strategy.

This tracking program was designed to flag all legislation and all regulation posted in official federal and state records. It was not designed to flag policy changes with a lesser degree of public notice, such as changes to preferred drug lists, prior authorization criteria, or dosing limits imposed by administrators of state Medicaid programs. Consequently, it was necessary to look elsewhere for these types of policies that influence opioid prescribing. Information regarding these types of changes to state Medicaid policies was gathered from a Kaiser Family Foundation survey of Medicaid programs. ${ }^{15}$ It should be noted that this survey covered only fiscal years 2016 and 2017. Thus, it is possible that additional, similar, changes made in 2018 were missed.

In addition, some policies were identified on the basis of information received from key informants, such as policy advocacy staff of other professional associations, and reviews of official state government and state licensing board websites.

While there is a difference between a rule (mandatory) and a guideline (advisory), we recognized that these terms have often been used interchangeably requiring us to scrutinize the data further to ensure the rule or guideline was classified correctly. Moreover, because professional association guidelines are, by definition, promulgated by nongovernmental organizations, they were not counted unless a state or federal authority had officially adopted them as a guideline or rule.

\section{CE Requirements}

FDA further requested that RPC enumerate CE requirements by state licensing boards, related to pain or safe opioid prescribing, during the 10 months following the release of the 2018 FDA Blueprint. FDA additionally requested information regarding the number of prescribers affected by these requirements, the extent to which a $\mathrm{CE}$ program complying with the Blueprint would meet those state requirements, and the number of $\mathrm{CE}$ programs related to opioid prescribing and opioid use disorder treatment available to prescribers during the designated time frame and the year prior.

These data were gathered in July 2019 by accessing websites of state licensing boards, state and national organizations representing the professions associated with prescribers, and independent $\mathrm{CE}$ organizations whose posted information included lists of state CE requirements. To help reduce potential error stemming from the inaccurate reporting of state $\mathrm{CE}$ requirements, we also employed open Internet searches involving "opioid continuing education," "pain continuing education," "controlled substances continuing education," and "opioid REMS continuing education." This exhaustive search strategy revealed that the information on several state licensing board websites was not current. We conducted additional e-mail and telephone follow-up with each individual licensing board, to determine the numbers of affected prescribers and to confirm the accuracy of our information regarding CE requirements. Remarkably, most licensing boards could not provide information on the number of their licensees who needed to complete their CE requirements. Consequently, the United States Department of Labor's Bureau of Labor Statistics Occupational Employment Statistics website was also used as a supplementary source to estimate the number of licensed prescribers.

To assess the degree to which a CE program adhering to the FDA educational blueprint would meet each state's $\mathrm{CE}$ requirements in pain and safe opioid use, we focused on the 10 bulleted knowledge requirements that were highlighted in FDA's 2018 REMS Blueprint: ${ }^{14}$

1. The fundamental concepts of pain management, including definitions and mechanisms of pain;

2. How to assess patients in pain, identifying risk factors for abuse and addiction; 
3. The range of therapeutic options for managing pain, including nonpharmacologic approaches and pharmacologic (non-opioid and opioid analgesics) therapies;

4. How to integrate opioid analgesics into a pain treatment plan individualized to the needs of the patient;

5. How to safely and effectively manage patients on opioid analgesics in the acute and chronic pain settings, including initiating therapy, titrating, and discontinuing use of opioid analgesics;

6. How to counsel patients and caregivers about the safe use of opioid analgesics, including proper storage and disposal;

7. How to counsel patients and caregivers about the use of naloxone for opioid overdose;

8. When referral to a pain specialist is appropriate;

9. The fundamental elements of addiction medicine;

10. How to identify and manage patients with opioid use disorder.

To conduct the assessment, two individual reviewers experienced with $\mathrm{CE}$ and opioid analgesic policy analyzed each pain/opioid $\mathrm{CE}$ requirement in all 50 states and the District of Columbia, comparing those requirements to the 10 knowledge requirements contained in the Blueprint. Each reviewer (RT, KD) performed the assessment independently using the following criteria:

- If all the state's content requirements were contained in the 2018 REMS Blueprint, the requirement was rated "meets", indicating that CE programs derived solely from the REMS Blueprint could meet the state requirements.

- If some, but not all, of the state's content requirements were contained in the 2018 REMS Blueprint, the requirement was rated "partially meets", indicating that $\mathrm{CE}$ programs derived solely from the REMS Blueprint could only partially meet the state requirements.

- Finally, if none of the state's content requirements were contained in the 2018 REMS Blueprint, the requirement was rated "does not meet", indicating that CE programs derived solely from the REMS Blueprint could not meet the state requirements.

In the event of disagreement, reviewers discussed and resolved any differences by consensus. It is important to note that the reviewers only focused on a comparison of
CE content with the Blueprint, not the length of the course or the time involved. Many states require only completion of a set number of $\mathrm{CE}$ hours or credits, with only general reference to pain or safe opioid prescribing.

Finally, to identify available CE programs on pain or safe opioid prescribing, open Internet searches using the terms "opioid continuing education," "pain continuing education," and "opioid REMS continuing education" were carried out. Programs identified by these searches were examined to determine if they were available during the assessment window, and those meeting that criterion were listed and counted.

\section{Results \\ State and Federal OA Policy Changes, 2016-2018}

Five-hundred twenty-seven (527) policies related to opioid prescribing were adopted from 2016 to 2018 (171 new policies in 2016; 201 in 2017; and 155 in 2018). Only 15 of the 527 policies $(2.8 \%)$ were federal policies, reflecting the extent to which opioid prescribing policy is regulated at the state level. OA policies were initially grouped into 24 descriptive categories. An illustration of the 10 most encountered policy issue areas is presented in Figure 1. Of the 527 policies, the most common policy changes were those relating to prescription drug monitoring programs (PDMPs) (246, 47\%); limiting opioid prescriptions in some way, including 34 directly limiting opioids in cases of chronic pain, 52 limiting opioids in the acute setting, and 19 limiting initial prescriptions (170, 32\%); and Medicaid policies (105, 20\%), illustrating that even when states do not directly limit opioids via statute or by licensing board rule, they can still substantially limit opioids through federal programs such as Medicaid. Additionally, 89 (17\%) policies established treatment guidelines and $70(13 \%)$ established standards for HCP education. State-level policies dominated each category, with only the practitioner education $(11.4 \%)$ and research (11.9\%) categories exceeding $10 \%$ of policies coming from the federal level.

Although some policies could arguably be assigned to more than one category due to their broad content, the organizational scheme helps to highlight and illustrate the emphasis of state and federal policy interventions during the study period. Accordingly, those summary results are reported here. Detailed policy information can be found in the Supplemental E-Table, Appendix B, that is associated with this article.

In addition, we found 35 policies across 25 states and the District of Columbia that directly referred to and/or 


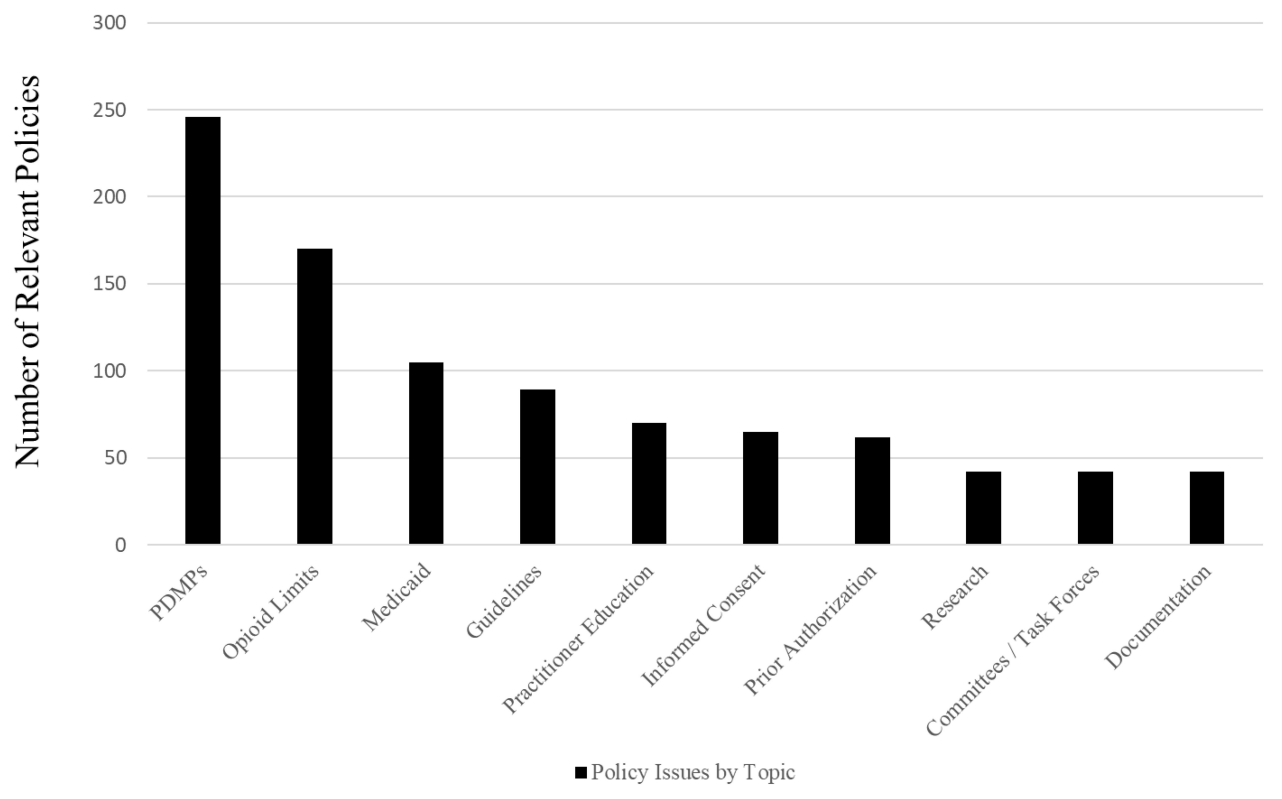

Figure I Number of policies in the ten most commonly encountered policy topic areas.

incorporated the 2016 CDC Guideline ${ }^{16}$ by reference. Given that the CDC Guideline was not released until March 2016, severely limiting opportunities to include it in that year's legislation, this represents rapid uptake of the Guideline by policymakers.

\section{State CE Policy, Number of Prescribers, and a Comparison of FDA's Educational Blueprint to State CE Programs}

In addition to the 70 policy changes noted above as affecting $\mathrm{HCP}$ education, FDA was interested in state $\mathrm{CE}$ requirements focusing on pain or safe opioid prescribing following the release of their REMS educational blueprint in 2018 and the number of available CE programs on these topics. Accordingly, those summary results are reported here. Detailed state CE policy information, Appendices CJ, can be found in the Supplemental E-Tables associated with this article.

States That Require Prescribers to Complete CE in Either Pain or Safe Opioid Prescribing as a Condition of Their Licensing or Renewal and the Number of Prescribers Affected

We examined eight categories of prescribers involving a total estimated population of 1.7 million. The term "prescribers" encompasses several distinct groups, including medical doctors, doctors of osteopathy, physician assistants, advanced practice nurses, podiatrists, naturopathic physicians, dentists, pharmacists, and optometrists, each of which may have a scope of practice including opioid prescribing.

Table 1 provides a concise picture of the CE requirement landscape across all eight prescriber categories along with the total number of estimated prescribers within each category in each state. In Table 1 , each cell contains the total number of prescribers of each type with pain or opioid prescribing $\mathrm{CE}$ requirements in each state; cells containing "No Req" are state-profession pairs where no requirements were found, while "Not Lic" indicates states that do not license naturopathic physicians. We found that some form of pain/opioid CE is required for at least one discipline in nearly every state, often across most licensed disciplines within the same state. Five states require $\mathrm{CE}$ for every prescribing profession licensed in that state (Arizona, Florida, New Jersey, Vermont, and West Virginia). Only four states require no pain/opioid CE of any kind (Colorado, Kansas, Missouri, and South Dakota).

Excluding nurses, our data revealed that in nearly three-quarters of all cases (128/172, 74.4\%), pain or safe opioid prescribing requirements mandate 3.5 hours or less of topic area $\mathrm{CE}$ per license renewal period. Eight states impose physician $\mathrm{CE}$ requirements only for pain specialists and/or those employed at a pain management clinic, while six states (California, Georgia, Louisiana, Oregon, Rhode Island, and Washington) have one-time pain- and opioid-related CE requirements that either are 


\begin{tabular}{|c|c|c|c|c|c|c|c|c|c|c|c|c|c|c|c|c|c|c|c|c|}
\hline 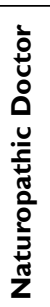 & $\begin{array}{l}\breve{u} \\
\stackrel{0}{Z}\end{array}$ & $\begin{array}{l}\text { J } \\
\propto \\
\text { ○े }\end{array}$ & $\underset{\text { O̊ }}{-}$ & $\begin{array}{l}\stackrel{u}{u} \\
\stackrel{0}{z}\end{array}$ & $\frac{n}{\alpha}$ & $\begin{array}{l}\mathbb{g} \\
\propto \\
\mathcal{Z}\end{array}$ & $\begin{array}{l}\text { ठ } \\
\propto \\
\stackrel{0}{0} \\
z\end{array}$ & $\begin{array}{l}\stackrel{u}{\exists} \\
\stackrel{0}{Z}\end{array}$ & $\begin{array}{l}\mathbb{g} \\
\stackrel{\alpha}{0} \\
\stackrel{0}{Z}\end{array}$ & $\begin{array}{l}\stackrel{u}{\exists} \\
\stackrel{0}{Z}\end{array}$ & $\begin{array}{l}\stackrel{u}{J} \\
\stackrel{\mathrm{o}}{z}\end{array}$ & 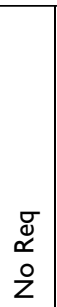 & $\begin{array}{l}\mathscr{J} \\
\propto \\
\stackrel{0}{Z}\end{array}$ & $\begin{array}{l}\stackrel{u}{Z} \\
\stackrel{\circ}{z}\end{array}$ & $\begin{array}{l}\stackrel{.}{u} \\
\stackrel{\Delta}{z}\end{array}$ & $\begin{array}{l}\stackrel{u}{J} \\
\stackrel{0}{0}\end{array}$ & 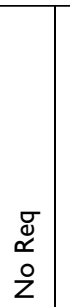 & $\begin{array}{l}\stackrel{u}{J} \\
\stackrel{\Delta}{Z}\end{array}$ & $\begin{array}{l}: \underline{U} \\
\breve{o} \\
z\end{array}$ & $\begin{array}{l}\text { g } \\
\stackrel{\alpha}{0} \\
\text { zo }\end{array}$ \\
\hline 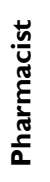 & $\begin{array}{l}\text { J } \\
\propto \\
\stackrel{o}{Z}\end{array}$ & 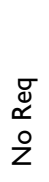 & $\stackrel{\sim}{\infty}$ & 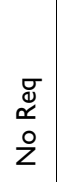 & $\begin{array}{l}\text { J } \\
\propto \\
\text { o }\end{array}$ & $\begin{array}{l}\text { g } \\
\propto \\
\text { Oे }\end{array}$ & $\begin{array}{l}\text { ष् } \\
\stackrel{2}{0} \\
\text { zo }\end{array}$ & ㅎㅇㅇ & 옹 & $\frac{\hat{n}}{\frac{m}{m}}$ & $\begin{array}{l}\mathbb{g} \\
\stackrel{\Xi}{0} \\
\stackrel{0}{z}\end{array}$ & 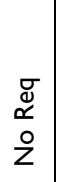 & 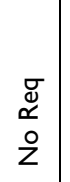 & $\begin{array}{l}\mathbb{g} \\
\stackrel{\alpha}{0} \\
\stackrel{0}{z}\end{array}$ & $\begin{array}{l}\mathscr{\Xi} \\
\propto \\
o \\
z\end{array}$ & 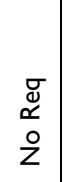 & 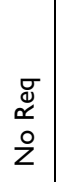 & $\begin{array}{l}\mathbb{J} \\
\propto \mathscr{q} \\
0 \\
\dot{Z}\end{array}$ & $\begin{array}{l}\text { J } \\
\stackrel{\mathscr{Y}}{0} \\
\text { O }\end{array}$ & 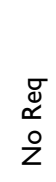 \\
\hline 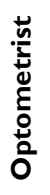 & 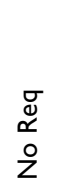 & $n$ & $\begin{array}{l}0 \\
\stackrel{8}{0} \\
\end{array}$ & 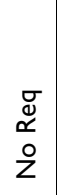 & 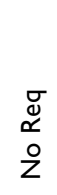 & $\begin{array}{l}\text { g } \\
\stackrel{0}{0} \\
\text { O }\end{array}$ & $\begin{array}{l}\mathbb{\Xi} \\
\stackrel{\not}{\circ} \\
\stackrel{0}{z}\end{array}$ & $\begin{array}{l}\text { J } \\
\stackrel{\leftrightarrow}{0} \\
\stackrel{0}{Z}\end{array}$ & 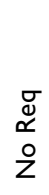 & \& & 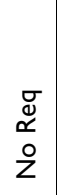 & 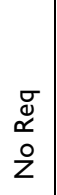 & $\begin{array}{l}\mathbb{g} \\
\stackrel{\Delta}{ } \\
\stackrel{0}{z}\end{array}$ & $\begin{array}{l}\mathbb{g} \\
\stackrel{\not}{\circ} \\
\stackrel{0}{z}\end{array}$ & $\begin{array}{l}\mathbb{g} \\
\stackrel{x}{0} \\
0 \\
z\end{array}$ & $\begin{array}{l}\mathbb{J} \\
\stackrel{\mathscr{C}}{0} \\
\stackrel{0}{Z}\end{array}$ & 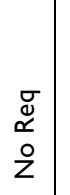 & $\underset{m}{\stackrel{m}{m}}$ & $\begin{array}{l}\text { g } \\
\stackrel{\mathscr{Q}}{0} \\
\stackrel{0}{Z}\end{array}$ & $\begin{array}{l}\mathscr{J} \\
\stackrel{\mathscr{Q}}{\circ} \\
\stackrel{0}{Z}\end{array}$ \\
\hline 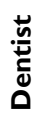 & $\underline{\underline{0}}$ & $\stackrel{\infty}{\wedge}$ & 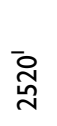 & $\begin{array}{l}\text { ğ } \\
\propto \\
\text { zo }\end{array}$ & 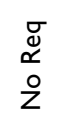 & 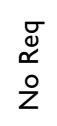 & 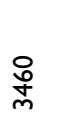 & $\begin{array}{l}\sigma \\
\stackrel{\mathscr{O}}{ } \\
\text { O }\end{array}$ & 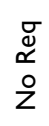 & 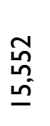 & 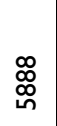 & 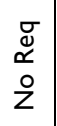 & 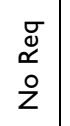 & $\begin{array}{l}\stackrel{\square}{\mathscr{y}} \\
\stackrel{0}{\circ} \\
\stackrel{0}{0}\end{array}$ & $\begin{array}{l}\mathbb{g} \\
\stackrel{\alpha}{\circ} \\
\stackrel{0}{z}\end{array}$ & $\stackrel{\infty}{\stackrel{0}{N}}$ & $\begin{array}{l}\text { J } \\
\propto \\
\text { Z }\end{array}$ & $\begin{array}{l}\stackrel{a}{\circ} \\
\text { o్m }\end{array}$ & 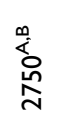 & 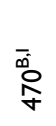 \\
\hline
\end{tabular}

\begin{tabular}{|c|c|c|c|c|c|c|c|c|c|c|c|c|c|c|c|c|c|c|c|c|}
\hline & 密 & 竞 & 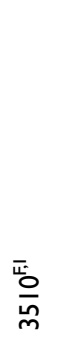 & $\begin{array}{l}\overline{0} \\
\stackrel{\delta}{\alpha}\end{array}$ & 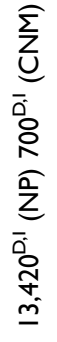 & $\begin{array}{l}\text { J } \\
\propto \\
0 \\
\dot{z}\end{array}$ & 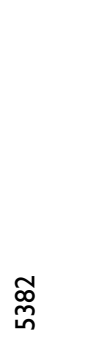 & 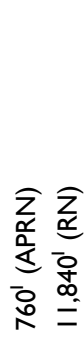 & 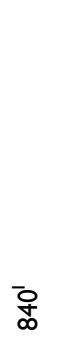 & $\frac{\frac{\infty}{m}}{\dot{m}}$ & $\begin{array}{l}\sigma \\
\stackrel{\Xi}{ } \\
\stackrel{0}{z}\end{array}$ & $\begin{array}{l}\overline{\mathrm{o}} \\
\overline{\mathrm{O}}\end{array}$ & 兑 & $\begin{array}{l}\text { 㔬 } \\
\text { hn }\end{array}$ & 品 & 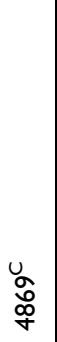 & $\begin{array}{l}\tilde{g} \\
\stackrel{o}{2} \\
o \\
z\end{array}$ & 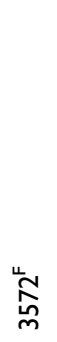 & 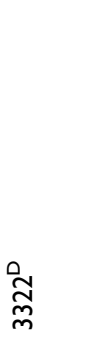 & 吕 \\
\hline & 织 & సे & శ్ণి & $\begin{array}{l}\sigma \\
\stackrel{\mathscr{O}}{ } \\
\stackrel{0}{Z}\end{array}$ & $\begin{array}{l}\text { J } \\
\propto \\
\stackrel{0}{z}\end{array}$ & 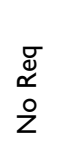 & 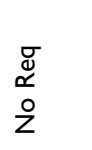 & $\begin{array}{l}\text { J } \\
\stackrel{\check{c}}{\circ} \\
\stackrel{0}{z}\end{array}$ & $\begin{array}{l}\sigma \\
\stackrel{\mathscr{J}}{ } \\
\stackrel{0}{Z}\end{array}$ & 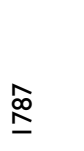 & $\begin{array}{l}\tilde{g} \\
\propto \\
\stackrel{o}{z}\end{array}$ & $\begin{array}{l}\square \\
\stackrel{\Xi}{~} \\
\circ \\
\stackrel{0}{Z}\end{array}$ & $\begin{array}{l}\text { J } \\
\propto \\
\stackrel{0}{Z}\end{array}$ & 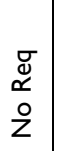 & $\begin{array}{l}\sigma \\
\stackrel{\Xi}{ } \\
o \\
\stackrel{z}{Z}\end{array}$ & 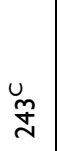 & $\begin{array}{l}\mathscr{g} \\
\stackrel{o}{2} \\
\circ \\
z\end{array}$ & 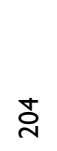 & $\stackrel{8}{ }$ & œ \\
\hline & م. & స్ర & $\frac{\overline{0}}{\mathrm{~N}}$ & $\overline{\bar{n}}$ & $\begin{array}{l}\text { J } \\
\stackrel{\mathscr{O}}{0} \\
\text { O }\end{array}$ & $\begin{array}{l}\text { J } \\
\stackrel{\Xi}{0} \\
\stackrel{0}{Z}\end{array}$ & 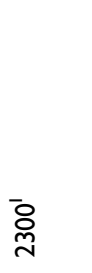 & $\begin{array}{l}\text { J } \\
\stackrel{\leftrightarrow}{0} \\
\text { o }\end{array}$ & $\begin{array}{l}\text { J } \\
\stackrel{\mathscr{O}}{0} \\
\stackrel{0}{Z}\end{array}$ & $\frac{\text { న̊ }}{2}$ & $\begin{array}{l}\text { J } \\
\stackrel{\mathscr{O}}{0} \\
\stackrel{0}{Z}\end{array}$ & 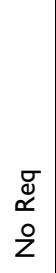 & $\begin{array}{l}\text { J } \\
\stackrel{\mathscr{O}}{0} \\
\stackrel{0}{Z}\end{array}$ & $\begin{array}{l}\mathbb{\Xi} \\
\stackrel{\alpha}{o} \\
\circ \\
z\end{array}$ & 总 & $\begin{array}{l}\text { Oo } \\
\dot{0}\end{array}$ & 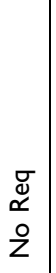 & 옹 & $\overline{\mathbf{I}}$ & 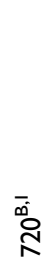 \\
\hline & 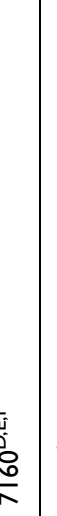 & 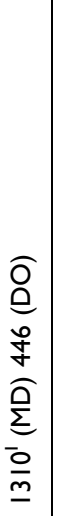 & $\begin{array}{l}\overline{0} \\
\text { O̊ } \\
\text { İ }\end{array}$ & 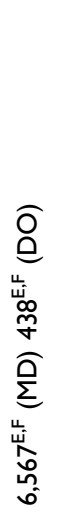 & $\frac{\bar{q}}{\frac{i}{f}}$ & $\begin{array}{l}\text { J } \\
\propto \\
\stackrel{0}{Z}\end{array}$ & 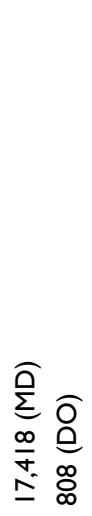 & $\begin{array}{l}\text { J } \\
\propto \\
\stackrel{o}{Z}\end{array}$ & $\begin{array}{l}\text { g } \\
\propto \\
\stackrel{0}{Z}\end{array}$ & 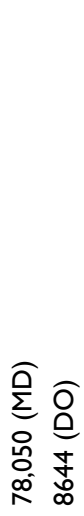 & 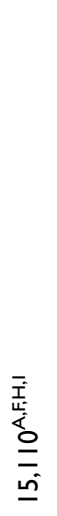 & $\begin{array}{l}\mathscr{J} \\
\propto \\
\stackrel{0}{z} \\
\end{array}$ & $\begin{array}{l}\text { J } \\
\propto \\
\stackrel{0}{Z}\end{array}$ & 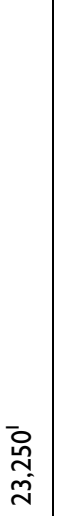 & $\begin{array}{l}\overline{8} \\
\pm \\
\dot{ \pm}\end{array}$ & 总 & 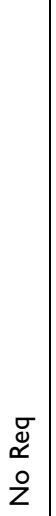 & '̄ & 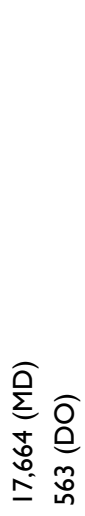 & $\begin{array}{l}\overline{\tilde{\omega}^{\prime}} \\
\text { ơ } \\
\text { d }\end{array}$ \\
\hline & $\vec{\psi}$ & $\stackrel{r}{<}$ & $\underset{<}{N}$ & $\frac{\alpha}{<}$ & $\mathbb{U}$ & U & $\mathfrak{u}$ & 㟔 & ด & $\vec{u}$ & త & $\overline{\mathbf{I}}$ & $\underline{\underline{a}}$ & $=$ & $\underline{\mathbf{z}}$ & $\leq$ & $\underline{\underline{v}}$ & $\grave{z}$ & $\unlhd$ & ए山 \\
\hline
\end{tabular}




\begin{tabular}{|c|c|c|c|c|c|c|c|c|c|c|c|c|c|c|c|c|c|c|}
\hline $\begin{array}{l}\text { J } \\
\propto \\
\text { O } \\
z\end{array}$ & 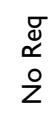 & $\begin{array}{l}\breve{u} \\
\stackrel{0}{u} \\
z\end{array}$ & 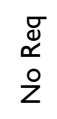 & $\begin{array}{l}. \breve{z} \\
\stackrel{\mathrm{o}}{z}\end{array}$ & $\begin{array}{l}\frac{U}{1} \\
\text { Z }\end{array}$ & $\begin{array}{l}\text { g् } \\
\stackrel{\alpha}{0} \\
\text { Z }\end{array}$ & $\begin{array}{l}. \breve{u} \\
\stackrel{0}{z}\end{array}$ & $\begin{array}{l}\frac{U}{1} \\
\text { Zn }\end{array}$ & $\begin{array}{l}\text { J } \\
\propto \\
\text { O̊ }\end{array}$ & $\begin{array}{l}\frac{U}{1} \\
\text { Zn }\end{array}$ & $\begin{array}{l}\text { J } \\
\propto x \\
\text { z }\end{array}$ & $\begin{array}{l}. \breve{u} \\
\stackrel{o}{z}\end{array}$ & 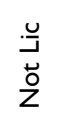 & $\begin{array}{l}\sigma \\
\dot{\Xi} \\
\propto \\
\circ \\
z\end{array}$ & $\begin{array}{l}\stackrel{.}{Z} \\
\stackrel{\Delta}{z}\end{array}$ & $\begin{array}{l}\stackrel{U}{Z} \\
\stackrel{0}{Z}\end{array}$ & 응 & $\begin{array}{l}\overline{\mathscr{U}} \\
\propto \\
\text { Oे }\end{array}$ \\
\hline $\begin{array}{l}\mathbb{g} \\
\propto \\
\text { o }\end{array}$ & 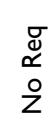 & 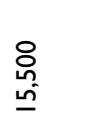 & $\begin{array}{l}\text { J } \\
\propto \mathscr{⿰} \\
\text { o }\end{array}$ & $\begin{array}{l}\mathscr{J} \\
\stackrel{x}{\circ} \\
\stackrel{0}{z}\end{array}$ & 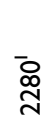 & 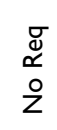 & $\begin{array}{l}\text { J } \\
\propto \\
\stackrel{0}{Z}\end{array}$ & $\begin{array}{l}\tilde{J} \\
\propto \mathscr{x} \\
\stackrel{0}{Z}\end{array}$ & $\begin{array}{l}\tilde{g} \\
\propto \\
\stackrel{0}{Z}\end{array}$ & $\begin{array}{l}\overline{\mathscr{D}} \\
\text { a }\end{array}$ & $\stackrel{\infty}{\circ}$ & $\begin{array}{l}\mathscr{\mathscr { Q }} \\
\propto \\
\stackrel{0}{Z}\end{array}$ & $\begin{array}{l}\mathbb{\mathscr { Q }} \\
\stackrel{1}{0} \\
\text { Z }\end{array}$ & 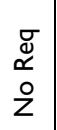 & $\begin{array}{l}\mathbb{g} \\
\propto \\
\stackrel{0}{z}\end{array}$ & $\begin{array}{l}\mathbb{J} \\
\propto \\
0 \\
\dot{Z}\end{array}$ & $\begin{array}{l}\overline{\text { Do }} \\
\text { r }\end{array}$ & 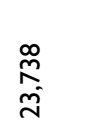 \\
\hline 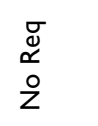 & $\begin{array}{l}\text { J } \\
\stackrel{\alpha}{\circ} \\
\stackrel{\circ}{Z}\end{array}$ & $\stackrel{m}{\underline{\underline{N}}}$ & চুষু & $\begin{array}{l}\mathbb{g} \\
\stackrel{\leftrightarrow}{0} \\
\stackrel{0}{z}\end{array}$ & $\begin{array}{l}\mathbb{J} \\
\propto \\
\\
\mathcal{Z}\end{array}$ & $\begin{array}{l}\mathbb{J} \\
\stackrel{\leftrightarrow}{~} \\
\text { O }\end{array}$ & $\begin{array}{l}\mathscr{J} \\
\propto \\
\stackrel{0}{Z}\end{array}$ & ন্ড & స్ & $\underline{\underline{0}}$ & ర్ల & 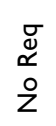 & 苦 & $\begin{array}{l}\mathbb{g} \\
\stackrel{\leftrightarrow}{\circ} \\
\stackrel{0}{z}\end{array}$ & $\begin{array}{l}\mathbb{g} \\
\check{x} \\
\stackrel{0}{Z}\end{array}$ & ర్లె & $\begin{array}{l}\mathbb{\sigma} \\
\propto \\
0 \\
Z\end{array}$ & : \\
\hline ్ㅗㄱ & $\begin{array}{l}\overline{\overline{0}} \\
\frac{0}{m} \\
\end{array}$ & $\frac{m}{\delta}$ & $\begin{array}{l}\text { 음 } \\
\underline{\Phi}\end{array}$ & 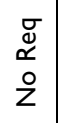 & $\frac{\mathfrak{O}}{\underline{0}}$ & $\begin{array}{l}\mathbb{g} \\
\stackrel{\alpha}{0} \\
\text { o }\end{array}$ & 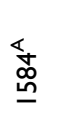 & స్ & ঃั & 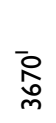 & 음 & $\begin{array}{l}\underset{\mathbb{N}}{\mathbb{N}} \\
\underline{\underline{0}}\end{array}$ & $\stackrel{\circ}{\infty}$ & $\begin{array}{l}\bar{g} \\
\stackrel{0}{o} \\
\circ\end{array}$ & $\begin{array}{l}\mathbb{g} \\
\stackrel{0}{\circ} \\
\dot{Z}\end{array}$ & $\begin{array}{l}\mathbb{g} \\
\propto \\
\dot{0} \\
z\end{array}$ & 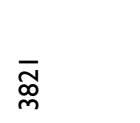 & $\frac{t}{\alpha}$ \\
\hline$\frac{\overline{\mathrm{o}}}{\frac{\mathrm{o}}{\mathrm{m}}}$ & 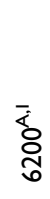 & 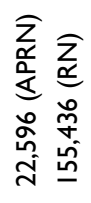 & 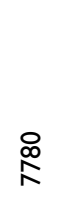 & $\begin{array}{l}\tilde{\sigma} \\
\propto \\
0 \\
\dot{z}\end{array}$ & 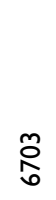 & $\begin{array}{l}\overline{0} \\
\text { ợ }\end{array}$ & 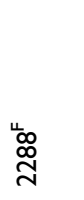 & $\begin{array}{l}\overline{\hat{0}} \\
\frac{0}{N}\end{array}$ & $\begin{array}{l}\overline{\underline{\sigma}} \\
\underline{\underline{\sigma}} \\
\underline{\underline{\sigma}}\end{array}$ & ৪্ㅎ & $\begin{array}{l}\overline{\widetilde{\varpi}} \\
\stackrel{\circ}{\alpha}\end{array}$ & $\begin{array}{l}\stackrel{0}{\underline{0}} \\
\underline{\underline{m}}\end{array}$ & $\begin{array}{l}\bar{\delta} \\
\text { ¿o } \\
\text { fo }\end{array}$ & $\stackrel{\mathfrak{\infty}}{=}$ & $\begin{array}{l}\stackrel{0}{2} \\
\text { o- }\end{array}$ & $\begin{array}{l}\overline{0} \\
0 \\
\underline{0}\end{array}$ & 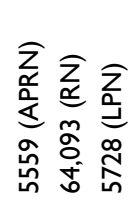 & $\begin{array}{l}\overline{0} \\
\stackrel{\infty}{\sim} \\
\stackrel{N}{N}\end{array}$ \\
\hline \&্ণ & $\underset{\llcorner}{\sharp}$ & \&̊ & 只 & 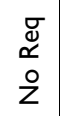 & 2 & $\begin{array}{l}\text { J } \\
\stackrel{x}{1} \\
\text { zo }\end{array}$ & ઠ & 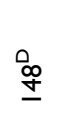 & $\begin{array}{l}\sigma \\
\stackrel{\Xi}{ } \\
\propto \\
\text { z }\end{array}$ & $\underset{\text { స్ }}{ }$ & $\stackrel{\circ}{\mathrm{m}}$ & $\stackrel{\substack{+\sim}}{ }$ & $\frac{\mathscr{n}}{\sigma}$ & 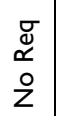 & $\begin{array}{l}\bar{g} \\
\stackrel{0}{o} \\
O \\
z\end{array}$ & 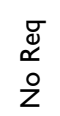 & $\underline{\underline{I}}$ & $\begin{array}{l}\text { రి } \\
\underline{0}\end{array}$ \\
\hline$\stackrel{\infty}{\stackrel{\infty}{N}}$ & $\begin{array}{l}\overline{\mathbf{X}} \\
\text { ్ㅗ }\end{array}$ & $\begin{array}{l}\text { J } \\
\propto \\
\circ \\
z\end{array}$ & $\begin{array}{l}\overline{0} \\
\stackrel{\circ}{\circ}\end{array}$ & 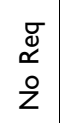 & ষ্ণ & $\begin{array}{l}\text { o } \\
\stackrel{\alpha}{0} \\
\text { Oे }\end{array}$ & $\begin{array}{l}\stackrel{\infty}{*} \\
\stackrel{\text { q }}{+}\end{array}$ & 웅 & $\underset{\infty}{\infty}$ & $\stackrel{\overline{0}}{\infty}$ & ర్థి & $\begin{array}{l}\overline{0} \\
\stackrel{0}{0} \\
\text { I }\end{array}$ & 흠 & 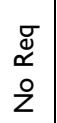 & $\begin{array}{l}\overline{0} \\
\text { y্z }\end{array}$ & 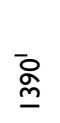 & $\frac{\pi}{N}$ & $\underset{\infty}{\sim \infty}$ \\
\hline 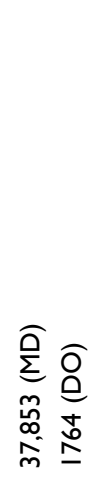 & 웅 & 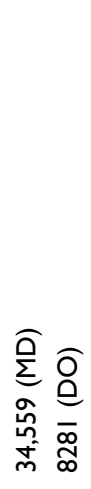 & $\begin{array}{l}0 \\
\infty \\
\tilde{F} \\
\underline{\tilde{L}}\end{array}$ & $\begin{array}{l}\mathbb{J} \\
\propto \\
0 \\
\dot{Z}\end{array}$ & 鿖 & $\begin{array}{l}\mathbb{J} \\
\stackrel{\leftrightarrow}{~} \\
\text { O }\end{array}$ & 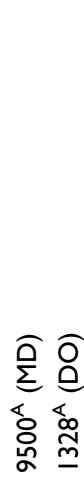 & 容 & 㝏 & $\begin{array}{l}\overline{8} \\
\text { d } \\
\text { i }\end{array}$ & 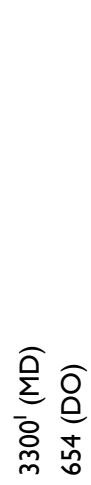 & $\frac{\bar{q}}{\sigma^{\circ}}$ & $\begin{array}{l}\overline{\dot{\delta}} \\
\frac{\Phi}{\alpha} \\
\underline{\alpha}\end{array}$ & $\begin{array}{l}\mathbb{g} \\
\stackrel{x}{ } \\
\stackrel{0}{z}\end{array}$ & $\begin{array}{l}\bar{w} \\
0 \\
o \\
\vdots \\
0 \\
0\end{array}$ & 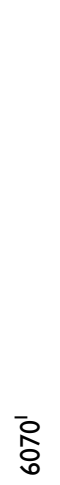 & 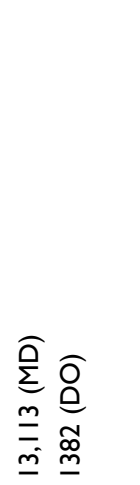 & 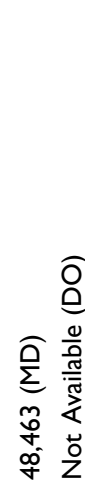 \\
\hline$\stackrel{0}{\Sigma}$ & $\Sigma$ & $\bar{\Sigma}$ & $\frac{Z}{\Sigma}$ & $\stackrel{\circ}{\Sigma}$ & $\sum^{n}$ & $\Sigma$ & 㞱 & $\vec{z}$ & $\frac{\mathrm{I}}{\mathrm{Z}}$ & $\bar{z}$ & $\sum_{Z}$ & $\grave{z}$ & $\mathrm{u}$ & $\mathrm{Q}$ & I & o & రิ & $\overleftarrow{a}$ \\
\hline
\end{tabular}




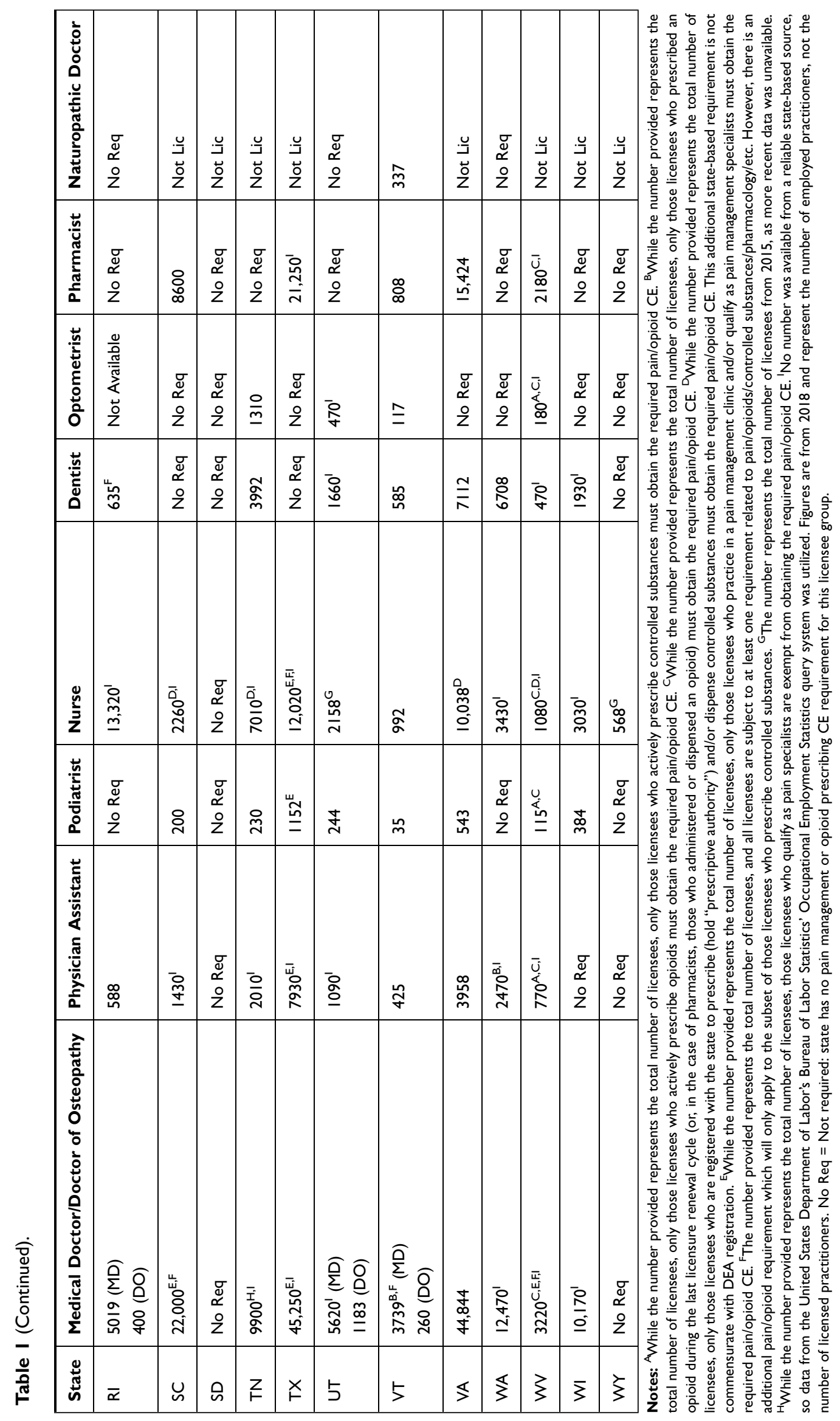


Table 2 Number of States, Including D.C., Where FDA Education Blueprint-Compliant CE Would Meet State CE Requirements

\begin{tabular}{|l|l|l|l|l|}
\hline & $\begin{array}{l}\text { Number of States } \\
\text { with Requirements } \\
\text { Met }\end{array}$ & $\begin{array}{l}\text { Number of States with } \\
\text { Requirements Partially } \\
\text { Met }\end{array}$ & $\begin{array}{l}\text { Number of States } \\
\text { with Requirements } \\
\text { Not Met }\end{array}$ & $\begin{array}{l}\text { Number of States with No } \\
\text { Requirements in Pain or } \\
\text { Opioid Rx }\end{array}$ \\
\hline MD/DO & $26(65 \%)$ & $10(25 \%)$ & $4(10 \%)$ & 11 \\
Nurse & $35(76 \%)$ & $5(11 \%)$ & $6(13 \%)$ & 5 \\
Pharmacist & $8(53 \%)$ & $2(13 \%)$ & $5(33 \%)$ & 36 \\
Dentist & $21(66 \%)$ & $6(19 \%)$ & $5(16 \%)$ & 19 \\
Physician Assistant & $23(66 \%)$ & $10(29 \%)$ & $2(6 \%)$ & 16 \\
Podiatrist & $22(76 \%)$ & $4(14 \%)$ & $2(10 \%)$ & 22 \\
Optometrist & $15(83 \%)$ & $1(6 \%)$ & $1(1 \%)$ & 33 \\
Naturopathic Physician & $1(25 \%)$ & $2(50 \%)$ & 47 \\
\hline
\end{tabular}

Notes: Percentages reflect the number of states/DC in each category versus the number of jurisdictions that had requirements for that profession.

prerequisites to initial licensure or are required of all licensees one time within a certain timeframe. For nonphysician prescribers, several states mandate topic area $\mathrm{CE}$ only for licensees who prescribe opioids or other controlled substances. Three states (New Mexico, Oregon, and Rhode Island) have instituted requirements that are very similar across all prescribing professions, and Utah mandates that all of the surveyed professions except pharmacists and naturopathic physicians complete a program every 2 years, with a requirement that such program must include all elements of the FDA Opioid REMS Blueprint, along with other topics.

CE requirements for nurses are substantially different, with 45 states and the District of Columbia requiring some or all types of nurses to accrue continuing education in pain management, substance use disorder, and/or safe opioid prescribing as a condition of license renewal. In seven cases, these requirements have been instituted despite nurses generally having no $\mathrm{CE}$ requirements for license renewal; note that five of those seven states require APRNs to maintain national certification, which may entail completing CE requirements. Twenty-seven states and the District of Columbia require $\mathrm{CE}$ in pharmacology for nurses with prescriptive authority. Often, these requirements are substantial, with many in the 10-to-15-hour range. Several of these states also specify that these CE hours must include content related to pain management, substance use disorders, or safe opioid use. Even though many of these requirements are solely focused on pharmacology, they have been retained in this analysis because an opioid REMS CE program would serve to partially fulfill that requirement.
The Degree to Which a CE Program Adhering to the FDA Educational Blueprint Would Meet Each State's CE Requirements

Although most states had already established their CE requirements by September 2018, the month the Blueprint was released and the current study period began, FDA was nevertheless interested in the degree to which the Blueprint would meet each state's $C E$ requirements in pain or safe opioid prescribing. Based on the assessment criteria we described earlier, we found that a CE program adhering to the FDA Blueprint would fully meet state CE requirements for $69 \%$ of state/profession pairs with such requirements. Of the 47 jurisdictions that required pain/opioid prescribing $\mathrm{CE}$, the most common reasons that the 10 bulleted knowledge requirements in the Education Blueprint would not fulfill state CE requirements, was due to states requiring the following topics that are not in the Blueprint: recommendations on dosage level and duration of prescribing, diversion training, PDMP education, and palliative care and/or end-of-life and/or discipline-specific education in those fields.

Table 2 summarizes the degree to which a CE program adhering to the FDA Blueprint, standing alone, would fulfill a state's CE requirements in pain or safe opioid prescribing.

\section{Availability of Pain/Safe Opioid Prescribing CE Programs}

Our searches identified 273 CE programs covering pain or safe opioid prescribing available to prescribers during the identified time frame. Sponsors of these programs included CDC (11 programs), continuing education provider companies (59 programs), JAMA journals (16 programs), national and state professional associations (96 programs), and universities, generally through their professional schools (91 programs); note 
that, in some cases, professional associations and universities partnered with continuing education provider companies, in which case we classified them as being offered by the association or university. While the search strategies used were comprehensive, it is likely that other programs were available, but were not identified. Additionally, there may have been programs available during earlier portions of the FDA-designated window that had closed and been removed from the Internet prior to our searches.

\section{Discussion}

Undertreated pain, prescription opioid misuse, and unintentional opioid overdose continue to pose significant challenges to policymakers and communities. These problems are large in scale, persistent, and complex. Moreover, they are also linked with mental health concerns and social determinants of health, creating a syndemic requiring multiple approaches to successfully mitigate what is arguably a moving target.

To achieve results, governments and non-governmental organizations have intervened in a variety of ways. Because the treatment of pain with OA has been touted as a contributor to the opioid overdose crisis, some interventions have focused on the safe and effective use of opioid therapy, including FDA's REMS-based educational efforts. This educational effort has produced a variety of outcomes including an FDA educational blueprint in 2018 that serves as a guide to REMS-based continuing educational efforts for opioid prescribers. Consonant with observed changes in patterns of misuse, what was once confined to ER/LA opioids has been expanded to include immediate-release/short-acting OAs. FDA also has made clear its intent that REMS-based opioid education should teach all healthcare providers caring for people with pain about non-pharmacological and non-opioid pharmacological pain treatments as well.

Following its release of the Blueprint in 2018, FDA wanted to better understand observed changes in OA prescribing patterns. Consequently, they asked RPC to provide information on major changes in OA policies at the state and federal levels from 2016 to 2018, as well as a summary of state CE policy in pain or safe opioid prescribing. We found that state and federal governments created 527 laws, rules, regulations, and guidelines concerning opioid therapy between 2016 and 2018, an average of 3.4 policies per year per state. The sheer volume of activity in this arena is testimony to the magnitude of policymakers' concerns about the opioid crisis, their recognition of its complexity, and their search for solutions.
The CDC Guideline, released in March 2016, proved especially influential to policymakers, as during the study period, 25 states directly referred to or incorporated it in 35 policies. Separately, states created 171 additional policies relating to limits on prescribing, dosing limits, and daily limits on supply, often based on recommendations in the Guideline but without specifically mentioning it. The extent to which these CDC Guideline-related policies remain in force or have been modified over the course of the next few years is a potential subject for further inquiry, especially in light of growing criticism regarding the translation of the Guideline's recommendations into laws and regulations, with potential deleterious impact on people with pain who are treated with opioids. Research into the impact of these policies on opioid prescribing also seems warranted, although we caution that separating their effects from those of the myriad other policies being adopted is a daunting task; further, the correlation between reduced opioid prescribing and positive or negative outcomes in people with pain is far from clear, and should be evaluated, as well.

Earlier research identified physician $\mathrm{CE}$ requirements related to pain management or safe opioid prescribing in five states $^{11}$ and 29 states. $^{12}$ By mid-2019, 40 states required such CE for physicians, while 46 states and the District of Columbia required some form of $\mathrm{CE}$ among a wide array of other prescriber professions, potentially affecting approximately 1.7 million prescribers. Policy changes related to $\mathrm{CE}$ were the fifth most common type of policies adopted by government agencies during the 2016-2018 study period. As might be expected, $\mathrm{CE}$ providers responded to these $\mathrm{CE}$ requirements, and offered more than 250 programs related to pain or opioid prescribing. While $\mathrm{CE}$ requirements were ubiquitous, we found a wide variation in specific $\mathrm{CE}$ content requirements among the states as well the type of prescriber that is affected. Subsequent research should examine the reasons for their variation, specific requirements, or omission of certain professionals.

While this article focused on state and federal policies created by governmental actors, private actors such as health insurance payors, pharmacy benefit managers, chain pharmacies, and healthcare systems, can create their own policies that can significantly impact the treatment of pain and the prevention of overdose and abuse nationwide. However, acquiring and evaluating those policies poses even greater challenges in terms of access, as these organizations consider the information to be proprietary. Further research to delineate the details of these types of policies should be carried out, in an effort to add to our 
understanding of observed changes in opioid prescribing in recent years.

\section{Study Limitations}

In conducting this study, we encountered barriers to acquiring data. Some states or agencies did not uniformly record the requested information, have it available, or respond to requests for information. The absence of a comprehensive listing or consistent data source often resulted in the necessity of seeking the same information from multiple sources to reduce the potential for error and missing data. It is possible that this caused us to miss some policies or CE requirements and programs. Information regarding the number of prescribers affected by $\mathrm{CE}$ requirements was especially challenging to acquire, requiring us to estimate that number in many states and producing data that are, at best, gross estimates.

\section{Conclusion}

Treating pain and preventing harm from prescription drugs will remain a significant challenge, a moving target with many potential remedies. Multiple efforts by multiple public and private actors are being made throughout the pain management, public health, and addiction communities. Many of the interventions we identified, including $\mathrm{CE}$, hold promise, but as with any intervention, it is essential that they be evaluated for their impacts and be informed by science. ${ }^{17-19}$ Given the complexity of the problem, the challenges associated with measurement, the role of illicit opioids, ${ }^{20}$ the multiple private actors, ${ }^{21,22}$ the challenge of tracking meaningful patient outcomes, and the sheer number of interventions across the United States, it will be difficult to determine the impact of a singular intervention in isolation or in combination with others. Despite the challenges associated with evaluating policy interventions in general, and $\mathrm{CE}$ in particular, ${ }^{23-26}$ we need to better understand how these interventions can positively influence prescriber behavior, reduce the harm associated with prescription drugs, and improve the treatment of pain.

\section{Disclosure}

This manuscript reports the results of an earlier study conducted by Ms. Duensing and Dr. Twillman under contract with the RPC. That study and this derivative article were funded solely by RPC. Ms. Duensing reports personal fees from REMS Program Companies, during the conduct of the study. Dr. Twillman is a consultant for Millennium Health and a speaker for Collegium Pharma. He also is the former Executive Director of the Academy of Integrative Pain
Management, which received financial support from a number of commercial interests, including opioid manufacturers. He also reports work was done under contract with the REMS Program Companies, administered by Syneos, during the conduct of the study; personal fees from Millennium Health, and from Collegium Pharma, and was formerly employed by Academy of Integrative Pain Management which received funding from numerous commercial interests, including opioid manufacturers, outside the submitted work. Dr. Ziegler provided writing services for this manuscript and has served as a paid consultant and speaker to government, industry, and NGOs on matters involving pain treatment, prescription drugs, and policy; and reports work was performed under contract in association with REMS Program Companies, administered by Syneos, during the conduct of the study; being the CEO of a private consulting firm, Drug \& Health Policy Consulting, LLC, a CME provider, speaker for Tarsus Medical/Painweek, a consultant CME for Global Education Group, Littleton, CO, a founder and director, volunteer, nonprofit organization for Center for Effective Regulatory Policy and Safe Access, Denver, CO, and a speaker for International Pelvic Pain Society, Chicago, IL, outside the submitted work. Dr M. Soledad Cepeda reports being a Johnson and Johnson stockholder, outside the submitted work. Drs. Cepeda and Kern are employees of Janssen Research \& Development an affiliate of Janssen Pharmaceuticals Inc., which markets several analgesic drug products including opioids. Dr. Wedin is an employee of Upsher-Smith Laboratories, LLC, a pharmaceutical company that markets prescription opioids. Dr. Salas is an employee of Daiichi Sankyo, Basking Ridge, NJ that marketed a prescription opioid and reports being an employee at DSI, during the conduct of the study.

\section{References}

1. Dahlhamer J, Lucas J, Zelaya C, et al. Prevalence of chronic pain and high-impact chronic pain among adults—United States, 2016. MMWR. 2018;67(36):1001-1006. doi:10.15585/mmwr.mm6736a2

2. Gladden RM, O'Donnell J, Mattson CL, Seth P. Changes in opioid-involved overdose deaths by opioid type and presence of benzodiazepines, cocaine, and methamphetamine - 25 States, July-December 2017 to January-June 2018. MMWR. 2019;68(34):737-744. doi:10.15585/mmwr.mm6834a2

3. Pain Relief Promotion Act (PRPA), H.R. 2260, 106th Congress (1999).

4. Brennan F. The US congressional "Decade on pain control and research" 2001-2011: a review. J Pain Pall Care Pharm. 2015;29:212-227.

5. Centers for Disease Control and Prevention National Center for Health Statistics. Wide-ranging online data for epidemiologic research (WONDER). Atlanta, GA; 2020. Available from http://wonder.cdc. gov. Accessed June 3, 2020.

6. MacLean CD, Fujii M, Ahern TP, et al. Impact of policy interventions on postoperative opioid prescribing. Pain Med. 2019;20 (6):1212-1218. doi:10.1093/pm/pny215 
7. Ziegler SJ. Governmental intervention in prescribing: reducing harm and medico-legal iatrogenesis. PWJ. 2013;1(3):36-40.

8. Ziegler SJ, Lovrich NP. Pain relief, prescription drugs, and prosecution: a four-state survey of chief prosecutors. J Law Med Ethics. 2003;31(1):75-100. doi:10.1111/j.1748-720X.2003.tb00060.x

9. Starr P. The Social Transformation of American Medicine: The Rise of a Sovereign Profession and the Making of a Vast Industry. New York, NY: Basic Books; 1982.

10. Schatman ME, Darnall BD. "Just saying no" to mandatory pain CME: how important is physician autonomy? Pain Med. 2013;14:1821-1825. doi:10.1111/pme.12285

11. Davis CS, Carr D. Physician continuing education to reduce opioid misuse, abuse, and overdose: many opportunities, few requirements. Drug Alcohol Depend. 2016;163:100-107. doi:10.1016/j. drugalcdep.2016.04.002

12. Xu J, Gribble A, Sigelman D. State CE requirements for physicians including requirements related to pain management and controlled substance prescribing. J Med Reg. 2017;103(3):12-20.

13. FDA REMS (2020). Food and drug administration, risk evaluation and mitigation strategies. Available from: https:/www.fda.gov/drugs/ drug-safety-and-availability/risk-evaluation-and-mitigation-strate gies-rems. Accessed June 3, 2020.

14. FDA education blueprint for health care providers involved in the treatment and monitoring of patients with pain (2018).Available from: https://www.fda.gov/drugs/information-drug-class/opioidanalgesic-risk-evaluation-and-mitigation-strategy-rems. Accessed April 6, 2020.

15. Smith VK, Gifford K, Ellis E, et al. Implementing coverage and payment initiatives: results from a 50-State Medicaid Budget Survey for State Fiscal Years 2016 and 2017. Available from: https://www.kff.org/medicaid/report/implementing-coverage-and-pay ment-initiatives-results-from-a-50-state-medicaid-budget-survey-forstate-fiscal-years-2016-and-2017. Accessed July 21, 2020.

16. Dowell D, Haegerich TM, Chou R. CDC guideline for prescribing opioids for chronic pain - United States, 2016. MMWR Recomm Rep. 2016;65(No.RR-1):1-49. doi:10.15585/mmwr.rr6501e1

17. Pew charitable trusts, results first initiative; 2020. Available from: https://www.pewtrusts.org/en/projects/results-first-initiative.

Accessed May 4, 2020.
18. Ziegler SJ. The proliferation of dosage thresholds in opioid prescribing policies and their potential to increase pain and opioid-related mortality. Pain Med. 2015;16(10):1851-1856. doi:10.1111/ pme. 12815

19. Organisation for Economic Co-operation and Development. Evaluating Laws and Regulations: The Case of the Chilean Chamber of Deputies. Paris: OECD Publishing; 2012. doi:10.1787/ 9789264176263-en

20. Haegerich TM, Paulozzi LJ, Manns BJ, Jones CM. What we know and don't know, about the impact of state policy and systems-level interventions on prescription drug overdose. Drug Alcohol Depend. 2014;145:34-47. doi:10.1016/j.drugalcdep.2014.10.001

21. Stoicea N, Costa A, Periel L, Uribe A, Weaver T, Bergese SD. Current perspectives on the opioid crisis in the US healthcare system: a comprehensive literature review. Medicine (Baltimore). 2019;98 (20):e15425. doi:10.1097/MD.0000000000015425

22. Schatman ME, Webster LR. The health insurance industry: perpetuating the opioid crisis through policies of cost-containment and profitability. J Pain Res. 2015;8:153-158. doi:10.2147/JPR.S83368

23. Heyward J, Olson L, Sharfstein JM, Stuart EA, Lurie P, Alexander GC. Evaluation of the extended-release/long-acting opioid prescribing risk evaluation and mitigation strategy program by the US food and drug administration: a review. JAMA Intern Med. 2020;180 (2):301-309. doi:10.1001/jamainternmed.2019.5459

24. Alford DP, Lazure P, Murray S, Hardesty I, Krause JR, White JL. National trends in prescription opioid risk mitigation practices: implications for prescriber education. Pain Med. 2019;20(5):907-915. doi: $10.1093 / \mathrm{pm} / \mathrm{pny} 298$

25. Barth KS, Guille C, McCauley J, Brady KT. Targeting practitioners: A review of guidelines, training, and policy in pain management. Drug Alcohol Depend. 2017;173:S22-S30. doi:10.1016/j. drugalcdep.2016.08.641

26. Price DW, Miller EK, Rahm AK, et al. Assessment of barriers to changing practice as CME outcomes. J Contin Educ Health Prof. 2010;30(4):237-245. doi:10.1002/chp.20088
Journal of Pain Research

\section{Publish your work in this journal}

The Journal of Pain Research is an international, peer reviewed, open access, online journal that welcomes laboratory and clinical findings in the fields of pain research and the prevention and management of pain. Original research, reviews, symposium reports, hypothesis formation and commentaries are all considered for publication. The manuscript management system is completely online and includes a very quick and fair peer-review system, which is all easy to use. Visit http:// www.dovepress.com/testimonials.php to read real quotes from published authors. 